\title{
Centrality analysis of metro network, a case study of Paris
}

\author{
Xi Wang ${ }^{1,2, a}$, Qingming Zhan ${ }^{1, b}$, Philippe Bonnin ${ }^{2, c}$ and Stephane Douady ${ }^{3, d}$ \\ ${ }^{1}$ School of Urban Design, Wuhan University, 430072 Wuhan, China \\ ${ }^{2}$ Labrotary AUS, 15 rue du seminaire de conflans, 94220 Charenton le Pont, France \\ ${ }^{3}$ Laboratory MSC, 10 rue Alice Domon et Leonie Duquet 75205 Paris, France \\ axiwang4224@gmail.com, bqmzhan@whu.edu.cn, philippe.a.bonnin@wanadoo.fr, \\ dstephane.douady@univ-paris-diderot.fr
}

Keywords: Metro network, Centrality, Structuality, traffic, Graph theory.

Abstract. Metro systems are playing an increasingly important role in the cities all around the world, due to the demand of reduction on traffic congestions and energy consumption. The purpose of the present study is to investigate the relationship between the entering traffic and a new conception of centrality Structuality on metro network. The centrality analysis is carried out for study the metro network in Paris. It is found that the coefficient of centrality could help indicate the potential traffic flow. The intersections on metro networks showed a relatively high centrality as well as entering traffic. An "effect of pump" could be observed around the intersections. This method could be applied to compare different network design in the planning phrase, as well as optimize the current metro networks.

\section{Introduction}

The world today is faced with the challenges that arise from global warming and the demand for sustainable development. In developing countries, due to rapid urbanization, an explosion in energy consumption is caused by a transition towards a car-oriented and large-scale urban morphology. To address this issue, the extension and application of metro system is increasingly necessary. For example, the metro networks altogether in 16 Chinese cities grew from 117 kilometers in 2000 to 1,755 kilometers in 2012. Furthermore, metro systems in 20 other Chinese cities have been approved or are currently under construction, and the total length in operation is about to reach 6,000 kilometers by 2020 [1].

It is important to understand the revolutionary significance of centrality in a metro system. By applying the centrality analysis to the metro stations, the distribution of passenger flux could be observed. Moreover, it could be adopted to evaluate the passenger flux of a new metro line; therefore the design of a network could be more adapted to the needs. After all, the objective of public transport is not just to transport the passengers from departure points to destinations, but it is also to realize the trip with less transfers and travel time.

The centrality of networks is a conception that first appeared in social science in the 1950s for evaluating the power of a person in the social network [2]. In the graph theory and network analysis, the centrality of a vertex indicates its relative importance in a graph. Later on, the conception is adapted to analyse the spatial configuration, for example the importance of a room in a building, as well as the usage frequency of a road in the road network. Three of the classic indicators of centrality are Degree, Closeness and Betweeness.

The application of the graph theory to the public transport network appeared later in the 1980s and 1990s. The metro system is a good example of complex network with different properties. The application of centrality of the transport networks is mostly adopted in the study of robustness. The more recent research on the metro system applied the conception of Betweenness [3]. 


\section{Method and data}

The classic indicators of centrality consider normally the Euclidian distance. However, from Place A to Place $\mathrm{B}$, a person tends to choose the simpler route than the shorter one. P. Bonnin [4] indicates that in the representation of network and also in its oral transmission, people tend to minimise the number of turns that they have to make. For example, in order to explicate to an interlocutor who asks for direction, a resident would essentially show the simplest route, which means one that contains the least turns, even if it is not the path with the shortest Euclidian distance.

The centrality Structurality is based on the simplest distance, which contains the minimum turns to make. Introduced by T. Courtat [5], this indicator has been adopted to analyse the road networks in comparison with the classic indicators of Closeness and Straightness. The centrality Structurality offers a hierarchical point of view of a city while underlining its major roads or an ancient enclosure.

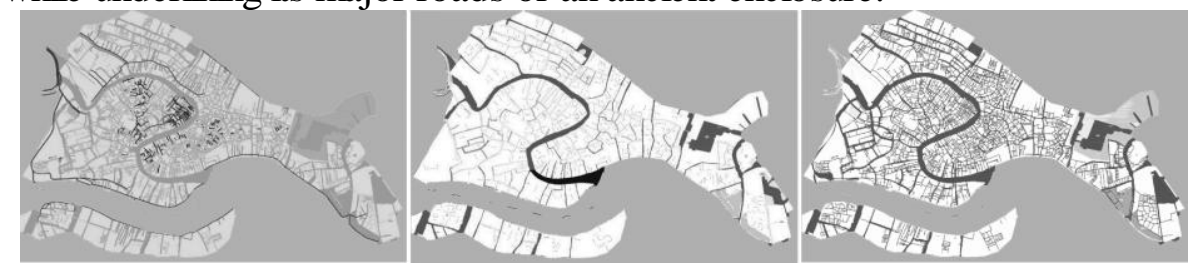

Figure 1 Structurality of the road network and canal network in Venice

For example, A. Perna [6] has adopted this indicator to analyse the networks in Venice. Fig. 1 presents the centrality of the road network (Left), and the canal network (Middle) and the two networks altogether (Right). It is difficult to find the logic of the centrality in the road network itself. However, it is much easier to understand the structure of the city if the canal networks or the two network systems altogether are considered. It shows that the structure of the city is constructed based on the fact that canals function as roads.

The centrality Structurality is more practical for studying the metro networks than the other classic centrality indicators, because the travel speed of metro is much faster than the vehicles on the roads when limited by traffic jam, therefore the Euclidian distance is far less important within the metro network. In this case, it is more important to choose a simple route with less transfer between metro lines.

The centrality of the metro station $\mathrm{M}_{0}, \mathrm{C}\left(\mathrm{M}_{0}\right)$, is calculated with the distance on number of transfers for going to all the other stations on the network. Because each station on the same line is reachable with the same number of transfers, only the distance for intersections would be distinguished, as $\operatorname{Order}\left(\operatorname{Line} / \mathrm{M}_{0}\right)$, and the number of stations on the line, as Nb.station.Line, so the formula is presented as Eq.1:

$$
\mathrm{C}\left(\mathrm{M}_{0}\right)=\sum_{\text {Line }} \operatorname{Order}\left(\text { Line } / \mathrm{M}_{0}\right) *(\mathrm{Nb} \text {.station.Line }) \text {. }
$$

There are two different cases (see Fig. 2). In the first case there are simple stations (non-intersection) on each line, in which case all the stations have the same centrality. The distances are calculated between this line and the others. In the second case for the intersections, the distance is calculated as distance between lines that cross at the intersections.
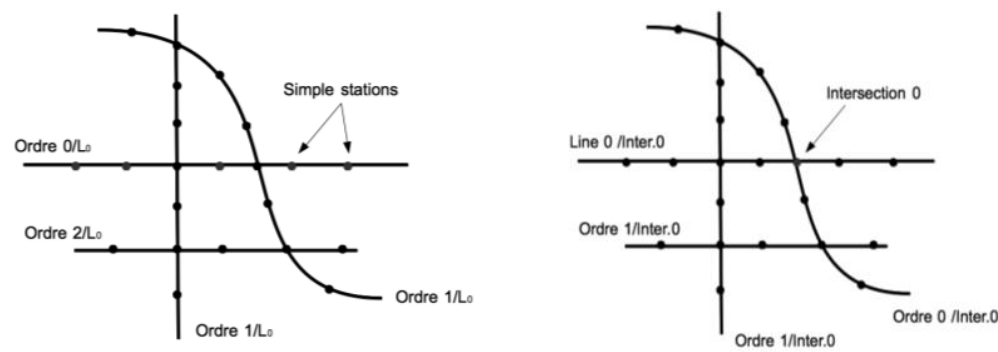

Figure 2 Two types of centrality Simplicity

The metro in Paris today is one of the most famous metro systems in the world, just like the metro in London and New York. The first metro line in Paris was built for serving the World Exposition in 1900. After 
more than 100 years' development, the metro system has significant impact on the city structure, the accessibility and the rhythm of people's daily activities. The 214 kilometers network is mostly built underground with 16 lines (including two minor lines $3 \mathrm{bis}$ and 7bis) and 303 stations. The GIS database of this study, as well as the annual entering traffic data in 2011, is created based on the data source from the Autonomous Operator of Parisian Transports [7].

\section{Result and discussion}

The centrality is the coefficient to evaluate the relative importance of a node in the network. It is therefore interesting to compare the correlation between the centrality and the traffic flow. In this section, comparisons are carried out first in a micro level on intersections. Then on a macro level, certain examples of metro lines are chosen to investigate their performances on centrality and traffic flow.

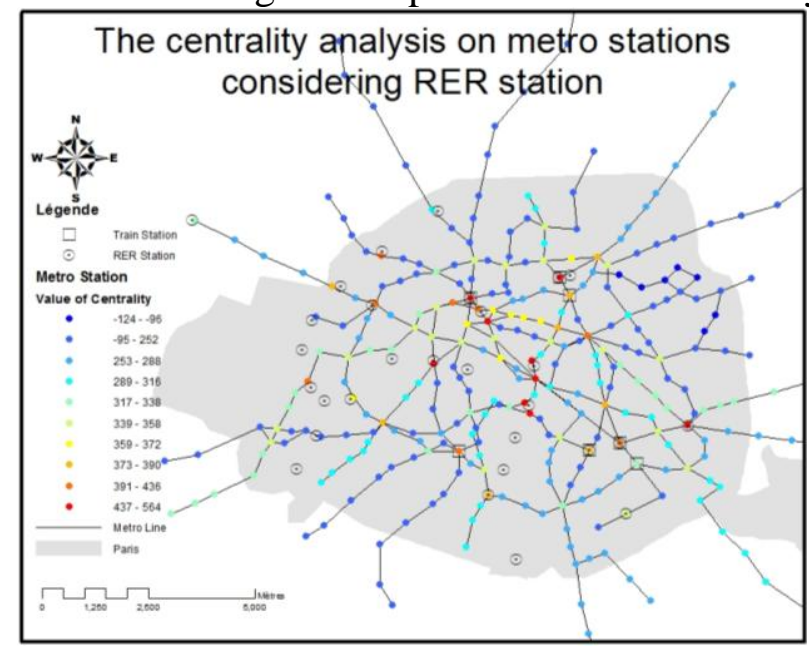

(a)

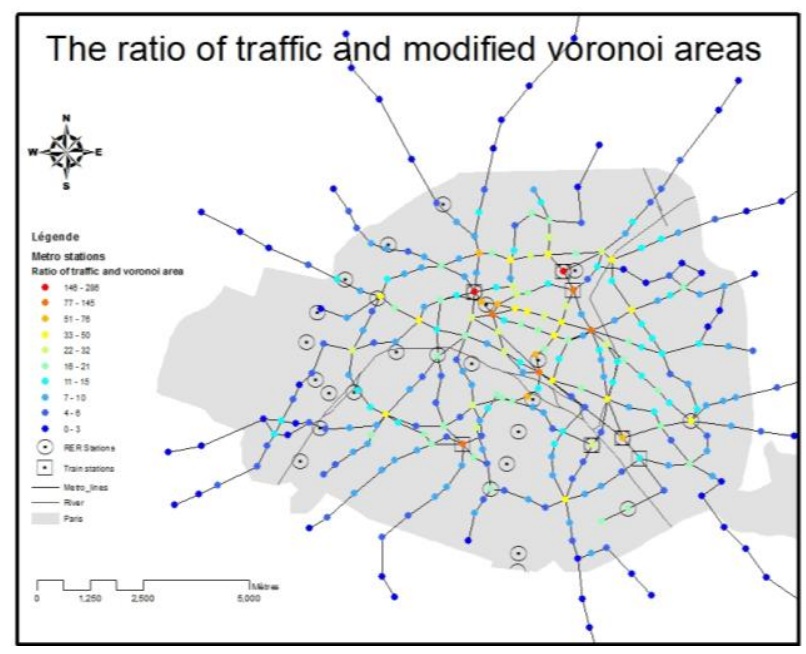

(b)

Figure 3. (a) Result of centrality analysis on metro stations; (b) traffic density on metro stations

In order to reduce the direct effect of various draining surface, the ratio of entering traffic flow and the Voronoi surface of metro stations is calculated as traffic density. Fig.3 shows that comparison between the coefficient of centrality and traffic density reveals a good coherency. Especially, it explains the particularity of intersection stations. In addition, there is an "effect of pump" near the intersections. For example, although there are several intersections around the three main metro stations Chatelet, Saint-Lazare and Nation, they still attract more traffic possibly because they have relatively higher centrality in the network.

There is also a good coherency between centrality Structuality and traffic on various metro lines. For example, Line 11 is a radial line starts in the city center by terminal Chatelet and continues to the suburb of Paris. Fig. 4 shows the centrality comparatively well and indicates the trend of traffic on line 11 . This line experiences a high traffic on one side of the line (Chatelet) situated in city center and a decrease on the other side in the suburb. The coefficient of relevance $\left(\mathrm{R}^{2}\right)$ between centrality and traffic is 0.747 , which is relatively high.

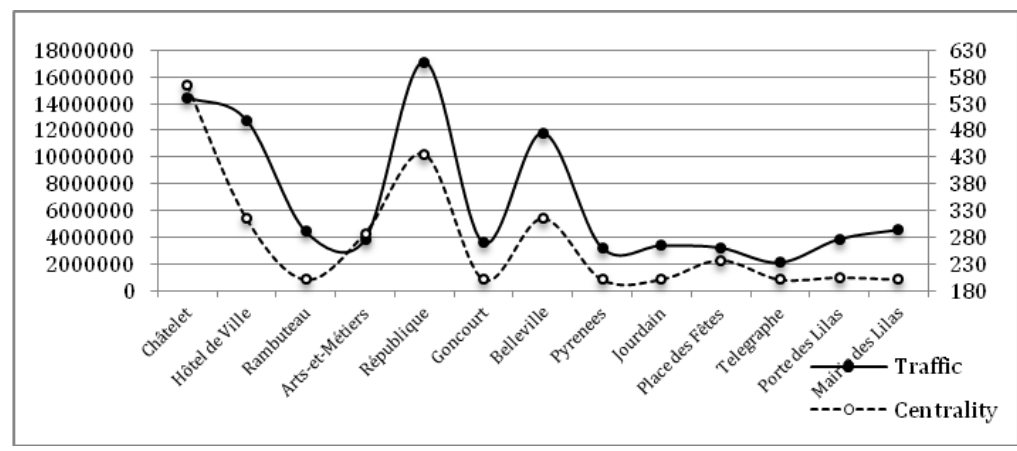

Figure 4 The traffic and centrality on Line 11 


\section{Conclusions}

By applying a newly developed concept of centrality Structuality, this study was designed to investigate the relationship between centrality of a metro network and its entering traffic. The centrality Structuality is based on the simplest distance, which contains the minimum turns to make. In the case of metro system, it refers to the distance with the least transfers between lines.

Centrality analysis of metro network in Paris indicates a good coherency between the centrality and the entering traffic. The results show that intersections are more significant than the simple station. Moreover, an "effect of pump" could be observed on most of the metro lines. The regression analysis reveals a strong correlation between the two indicators on various metro lines. It shows that the calculation of centrality could help to understand the passenger flux and their choices.

Overall, the coefficient of centrality could help to interpret the distribution of traffic flow in a metro system. It could be especially useful for the urban planners and decision makers to compare different scenarios of metro systems in the planning phase. In addition, it might also contribute to the optimization of an existing metro network. By adopting the analysis of centrality Structuality, further research can be carried out to understand how a metro network evolves structurally during its development.

\section{Acknowledgements}

This work was financially supported by the Chinese Scholarship Council (CSC), and developed in the context of research group Morphocity.

\section{References}

[1] Newman, M. E. J. (2010). Networks: An Introduction. Oxford, UK, Oxford University Press.

[2] Derrible, S. (2012). Network Centrality of Metro Systems. PLoS ONE 7(7): e40575.

[3] Bonnin, P. and S. Douady (2013). Morphogenèse du réseau viaire. Les réseaux dans le temps et dans l'espace.

[4] Courtat, T., S. Douady and C. Gloaguen (2011). Centrality maps and the analysis of city street networks. Proceedings of the 5th International ICST Conference on Performance Evaluation Methodologies and Tools. Paris, France, ICST (Institute for Computer Sciences, Social-Informatics and Telecommunications Engineering): 316-321.

[5] Perna, A., P. Kuntz and S. Douady (2011). Characterization of spatial network-like patterns from junction geometry. Physical Review E vol. 83(Issue 6).

[6] RATP. (2013). Plateforme opendata de la RATP. Retrieved January, 15, 2013, from http $/ /$ data.ratp.fr. 